\title{
Isolated twisted hematosalphinx misleading with ovarian cyst torsion
}

Vaibhav Khairnar*, Shalini Mahana Valecha, Pandeeswari

Department of Obstetrics and Gynecology, ESI-PGIMSR, Mumbai, Maharashtra, India

Received: 05 December 2018

Accepted: 05 February 2019

\section{*Correspondence:}

Dr. Vaibhav Khairnar,

E-mail: vaibhav.bjmc@gmail.com

Copyright: () the author(s), publisher and licensee Medip Academy. This is an open-access article distributed under the terms of the Creative Commons Attribution Non-Commercial License, which permits unrestricted non-commercial use, distribution, and reproduction in any medium, provided the original work is properly cited.

\begin{abstract}
Normal or chronically inflamed fallopian tube can undergo torsion and present as acute abdomen, simulating clinically as ectopic gestation. Torsion of the fallopian tube is less frequent but significant cause of lower abdominal pain in reproductive age women that is difficult to recognize preoperatively. Authors present a rare case of hematosalpinx with torsion at its pedicle with hemoperitonium who presented as 28 years old female with acute abdomen that was successfully treated. In cases presenting with hemoperitoneum diagnosis of ruptured ectopic pregnancy should be made unless proved otherwise during reproductive age. Rarely ruptured ovarian cyst may also be a cause. Unfortunately, hematosalpinx sometimes can undergo torsion due to circulatory imbalance and can present as hemoperitoneum and circulatory collapse due to rupture. There have been no specific symptoms, clinical findings, imaging or laboratory characteristics identified for this condition. Imaging findings are non-specific in the preoperative diagnosis of torsed fallopian tubes. Therefore, most of cases with isolated fallopian tubal torsion had a delayed diagnosis and a subsequent delay of timely intervention that may result in failure to save tubal function. Torsion of tube can lead to hematosalpinx, hemoperitonium and necrosis of tube which necessitates urgent surgical management. This case report describes a twisted hematosalpinx presentation without any predisposing high-risk factors. A tubal torsion should be suspected in females with acute pelvic pain, of any age group. An early diagnosis and treatment are required to prevent complications. This rare case may highlight a new insight into pathogenesis of tubal torsion associated with hematosalpinx.
\end{abstract}

Keywords: Acute abdomen, Hemoperitonium, Torsion hematosalpinx

\section{INTRODUCTION}

The uterine adnexa consist of the ovaries, the fallopian tubes, and the uterine ligaments. A hydrosalphinx may be unilateral or bilateral and is primarily a sequel of acute or chronic pelvic inflammatory disease. A hydrosalphinx usually is asymptomatic; however, it may be associated with chronic pelvic pain, dyspareunia, and a sense of pelvic pressure. Isolated torsion of the fallopian tube is an uncommon cause of acute lower abdominal pain. The incidence is estimated to be 1 in 500,000 women. It is often found in reproductive age women and is found less in prepubertal and perimenopausal women.

Acute abdomen is one of the important presentations in gynaecological practice, the common causes being acute pelvic inflammatory disease, torsion of ovarian cyst and ectopic pregnancy apart from surgical causes. Tubal pregnancy is an important diagnosis to be ruled out in any female patient presenting with acute abdomen, especially post sterilisation. Authors present a rare case of hematosalphinx with torsion at its pedicle with hemoperitoneum who presented with acute abdomen. ${ }^{1}$ 
Possible etiologies for the fallopian tube torsion are proposed as followed: anatomic abnormalities, such as long mesosalpinx, tortuous dilated tube (hydro- or hematosalpinx), tubal mass (tubal neoplasm) and adnexal mass (adjacent ovarian or paraovarian tumour), physiologic abnormalities, such as abnormal peristalsis or periovulatory spasm, hemodynamic abnormalities, such as adnexal venous congestion, sellheim theory (sudden body position changes), trauma, previous surgery or disease, such as pelvic inflammatory disease, pelvic adhesion and tubal ligation, gravid and enlarged uterus or uterine masses. $^{2}$

Hematosalphinx has been observed as an unusual complication after medical abortion with oral mifepristone with misoprostol. ${ }^{3}$ Hematosalpinx has been reported in cases of ectopic pregnancy, even bilateral ones in cases of unilateral ectopics., ${ }^{4,5}$ In cases of gynaecologic and obstetric disorders presenting with abdominal pain, it is seen that usually the torsion of the adnexa is at the pedicle between the ovary and the uterus in twisted ovarian cysts. ${ }^{6}$

\section{CASE REPORT}

A 28 years old mother of two children, (non tubectomised) with known case of hypothyroidism admitted with history of acute pain in left lower part of the abdomen of one day duration, severe in nature, continuous. Menstrual cycles were regular with normal flow and duration. She was in the third day of menstrual cycle; urine pregnancy test was negative.

On examination, her vitals were stable with pulse rate 98/min and systemic examination was within normal limits. Per abdomen examination showed tenderness in the left iliac fossa, no guarding or rigidity and bowel sounds were present.

Bimanual pelvic examination revealed normal sized uterus with tender cystic mass in left adnexa approximately $10 \quad \mathrm{X} \quad 10 \mathrm{~cm}$ and cervical motion tenderness was present.

Urgent Ultrasound suggestive of left sided cystic mass $9.5 \times 6.5 \times 3.4 \mathrm{~cm}$, left ovary appears to the part of the cystic lesion, moderate free fluid in pouch of dugles with echos. The uterus and right ovary were normal. Doppler findings showed compromised vascularity suggestive of torsion.

Her urine pregnancy test was negative, and all biochemical and hematological parameters were within normal limits. A diagnosis of twisted ovarian cyst was made, and the patient was shifted for emergency laparotomy.

At laparotomy, contrary to our preoperative diagnosis there was a left twisted hematosalphinx $(10 \times 8 \times 4 \mathrm{~cm})$ with hemoperitoneum $(100 \mathrm{cc})$. The left ovary was normal, and rest of the pelvic structures did not reveal any pathology. Left salpingectomy was done. On cut section of specimen clot of 50cc seen without any evidence of chorionic tissue. Post-operative period was uneventful and she was discharged on 4th post-operative day. Histopathology showed features consistent with hematosalphinx.

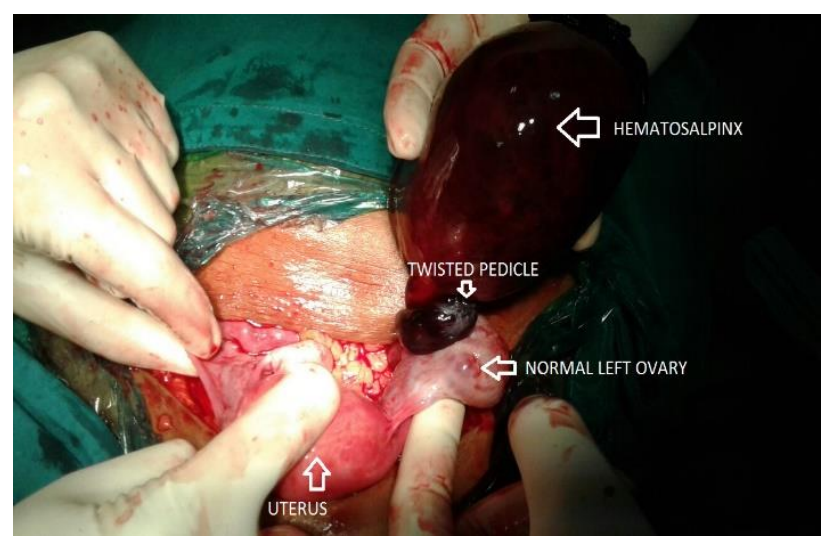

Figure 1: Intraoperative picture of twisted hematosalphinx.

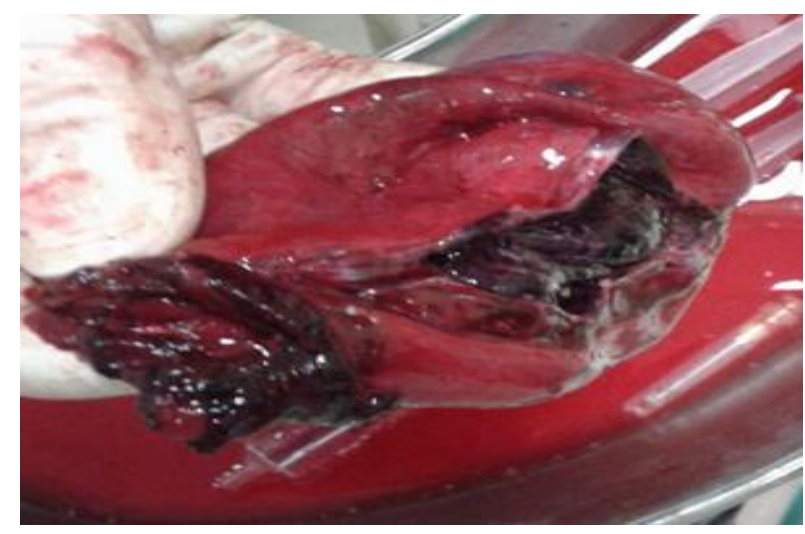

Figure 2: Drained hematosalphinx.

\section{DISCUSSION}

A number of causes may account for a hematosalphinx, by far the most common being a tubal pregnancy. It may also result from tubal disease such as inflammation of the fallopian tube. A hematosalphinx can also be associated with endometriosis or tubal carcinoma. Further, if menstrual blood flow is obstructed (crypto menorrhea), caused for instance by a transverse vaginal septum, and gets backed up it may lead to a hematosalphinx. A hematosalphinx from other conditions may be painless but could lead to uterine bleeding. Blood may also escape into the peritoneal cavity leading to a hemoperitoneum. In all of these cases, hematosalpinx can be very well diagnosed by gynaecologic ultrasound examination.

Regad surveyed 201 cases of fallopian tube torsion and found a normal fallopian tube in only $24 \%$ and quoted that the incidence of fallopian tube torsion is unknown. Imaging findings in torsion of the fallopian tube 
described so far are nonspecific, and clinical correlation is mandatory. ${ }^{7}$

Associated disease can be Tubal pregnancy (most common), Endometriosis, Fallopian tube cancer, Salpingitis, Obstructed vagina resulting in menstrual blood backflow into fallopian tubes (cryptomenorrhea). Unusual complication after medical abortion with oral mifepristone with misoprostol.

Torsion of normal tube in any age in childhood or adolescents is because of altered physiological mobility of organs. In reproductive age group there are many risk factors seen preceding to torsion like salpingitis, hydrosalpinx, hematosalpinx, ectopic pregnancy, ovarian tumours, adhesions or bands. ${ }^{8}$

\section{Complications}

Complication of a hematosalphinx may happen when part of the tube becomes twisted (torsion), which can result in death if not fixed. Fallopian tube torsion has an estimated prevalence of 1 in 1.5 million women. Isolated tubal torsion is a rare event in women of reproductive age and even more so in adolescent girls. An ectopic pregnancy carries with it the deadly risk of uterine tube rupture and resulting haemorrhage.

Risk factors of this situation are fallopian tube infection, advanced maternal age (the risk increases with age). Hematosalphinx may have no symptoms (asymptomatic), or the symptoms may be that of the condition with which it is associated: abdominal pain, vaginal spotting, and pelvic pain, uterine bleeding, fever and frequent urination.

\section{Investigations}

Temperature, white blood cell count, and erythrocyte sedimentation rate may be normal or slightly elevated. Imaging findings are non-specific in the preoperative diagnosis of torsed fallopian tubes. The ultrasound image associated with hydrosalphinx may reveal an elongated, convoluted cystic mass, tapering as it nears the uterine cornua and the ipsilateral ovary. Doppler evaluation could be helpful in a patient with a history of tubal ligation if high impedance or absence of flow in a tubular structure is noted. Computed tomography or magnetic resonance imaging is also reported to be helpful for diagnosis..$^{9,10}$

\section{Management}

Isolated tubal torsion can be managed with either detorsion or simple salpingectomy. Adnexal de-torsion has an extremely low risk of thromboembolic events. However, it should be performed as early as possible to avoid irreversible damage to the tissue. The operative approach could be conventional exploratory laparotomy or laparoscopic surgery. Several studies of adnexal torsion have demonstrated that the color, size, and degree of edema do not correlate with necrosis and subsequent return to normal tubal or ovarian function. Immediate detorsion is always recommended, because it is unclear how long a patient has until irreversible damage occurs. ${ }^{11}$

\section{Prevention}

Generally womanly immunity becomes weaker over menstrual period or after abortion and delivery. Therefore, it is time for females to keep the good habit of personal hygiene, in which way uterine tubes can be saved from invading of bacteria. ${ }^{12}$

\section{CONCLUSION}

In conclusion, authors suggest that in the differential diagnosis of lower abdominal pain in a reproductive age woman, torsion of the fallopian tube leading to hematosalpinx should be considered, although it is the low incidence. Imaging techniques may be suggestive but not conclusive as in our case misleading with ovarian cyst torsion. Expedient diagnosis is important to prevent adverse sequelae. However, the diagnosis can be challenging because the symptoms are relatively nonspecific.

\section{Funding: No funding sources \\ Conflict of interest: None declared \\ Ethical approval: Not required}

\section{REFERENCES}

1. Lakshmikantha G, Dwarakanath L, Kumari R. Torsion of hematosalpinx with hemoperitoneum. J Evolution Med Dental Sci. 2013;2(7):644-7.

2. Hansen O.H. Isolated torsion of the fallopian tube. Acta Obstet Gynecol Scand. 1970;49:3-6.

3. Lin SK, Ho ES, Chen YJ. Haematosalpinx: an unusual complication after medical abortion with oral mifepristone and misoprostol. Ultrasound Obstet Gynecol. 2005;25:416-7.

4. Atri M, de Stempel J, Bret PM. Accuracy of transvaginal ultrasonography for detection of haematosalpinx in ectopic pregnancy. $\mathrm{J}$ Clin Ultrasound. 1992;20:255-61.

5. Sindos M, Wang TF, Pisal N. Bilateral haematosalpinx in a case of ectopic pregnancy: a clinical dilemma. Am J Obstet Gynaecol. 2003;189:892-3.

6. Madhusudan Dey Lt Col, Reema Kumar Lt Col. Isolated torsion of hydrosalpinx. Med J Armed Forces India. 2013; 69(2):178-180.

7. Vijayaraghavan SB, Senthil S. Isolated Torsion of the Fallopian Tube. Journal of Ultrasound in Medicine.2009;28:657-2.

8. Dwivedi AD, Falleiro J, Debnath S. Torsion fallopian tube (A Case Report). Med J Armed Forces India. 1997;53(4):315. 
9. Ghossain MA, Buy JN, Bazot M, Haddad S, Guinet C, Malbec L, et al. CT in adnexal torsion with emphasis on tubal findings: correlation with US. J Computer Assisted Tomography.1994;18(4):619-25.

10. Terada Y, Murakami T, Nakamura SI, Sato Y, Niikura H, Ito K. Isolated torsion of the distal part of the fallopian tube in a premenarcheal 12-year-old girl: a case report. Tohoku J Experimental Med. 2004;202(3):239-43.

11. Lim SY, Park JC, Bae JG, Kim JI, Rhee JH. Isolated torsion of bilateral fallopian tubes combined with tubal endometriosis: A case report. Korean J Obstet Gynecol. 2012;55(1):55-58.

12. Wang PH, Yuan CC, Chao HT, Shu LP, Lai CR. Isolated tubal torsion managed laparoscopically. J Am Assoc Gynecol Laparosc. 2000;7(3):423e427.

Cite this article as: Khairnar V, Valecha SM, Pandeeswari. Isolated twisted hematosalphinx misleading with ovarian cyst torsion. Int J Reprod Contracept Obstet Gynecol 2019;8:1219-22. 